\title{
Hostility, depression and incident cardiac events in the GAZEL cohort
}

\section{Running title: Hostility, depression and cardiac events}

Nadine Hamieh ${ }^{1,}{ }^{*}$, Ph.D. student, Pierre Meneton ${ }^{2}$, Ph.D., Marie Zins ${ }^{3}$, M.D., Ph.D., Marcel Goldberg ${ }^{3}$, M.D., Ph.D., Emmanuel Wiernik ${ }^{4}$, Ph.D., Jean-Philippe Empana ${ }^{5}$, M.D., Ph.D., Frederic Limosin ${ }^{6}$, M.D., Ph.D., Maria Melchior ${ }^{1}$, Sc.D., Cedric Lemogne ${ }^{6,}$ M.D., Ph.D.

${ }^{1}$ Sorbonne Université, INSERM, Institut Pierre Louis d'Épidémiologie et de Santé Publique, IPLESP, Équipe de Recherche en Épidémiologie Sociale, F75012, Paris, France

${ }^{2}$ INSERM U1142 LIMICS, UMRS 1142, Sorbonne Universities, UPMC University of Paris 06, University of Paris 13, Paris, France

${ }^{3}$ Université de Paris, Faculty of Medicine, Paris, France; INSERM, Population-based Epidemiological Cohorts Unit, UMS 011, Villejuif, France

${ }^{4}$ INSERM, Population-based Epidemiological Cohorts Unit, UMS 011, Villejuif, France

${ }^{5}$ INSERM U970, Paris Cardiovascular Research Center, France; Université de Paris, Faculty of Medicine, Paris, France

${ }^{6}$ Université de Paris, INSERM, Institut de Psychiatrie et Neurosciences de Paris (IPNP), UMR_S1266, Paris; AP-HP.Centre-Université de Paris, Hôpital européen Georges-Pompidou, Service de psychiatrie et d'addictologie de l'adulte et du sujet âgé, Paris, France ; Université de Paris, Faculty of Medicine, Paris, France

* Corresponding author: Nadine Hamieh, PhD student Pierre Louis Institute for Epidemiology and Public Health (IPLESP/ INSERM UMR_S 1136) Department of Social Epidemiology (ERES) Faculty of Medicine, Saint-Antoine 27 rue de Chaligny 75012 Paris, France Email: nadine.hamieh@iplesp.upmc.fr Tel: +33 (0) 666883803 
Disclaimer: The views and opinions expressed in this report are those of the authors and should not be construed to represent the views of any of the sponsoring organizations, agencies, or the US government.

\section{Competing interests statement}

Cédric Lemogne declares grants, personal fees and non-financial support from Lundbeck, personal fees from Boehringer Ingelheim, personal fees from Janssen, non-financial support from Otsuka Pharmaceutical, outside the submitted work. For the remaining authors none were declared.

\section{Conflict of Interest}

Cédric Lemogne declares grants, personal fees and non-financial support from Lundbeck, personal fees from Boehringer Ingelheim, personal fees from Janssen, non-financial support from Otsuka Pharmaceutical, outside the submitted work. Frédéric Limosin declares personal fees and non-financial support from Lundbeck, and non-financial support from Janssen-Cilag and Otsuka Pharmaceutical, outside the submitted work. For the remaining authors none were declared. 


\section{Introduction}

Cardiovascular disease (CVD) is the first cause of death worldwide (World Health Organization, 2017, May 17). Beside the modifiable traditional CVD risk factors (obesity, hypertension, diabetes, hypercholesterolemia and smoking (Ezzati M et al., 2003; Ezzati M et al., 2004; Ezzati M et al., 2002; Ezzati et al., 2005; Tzoulaki et al., 2016)), several psychological characteristics are associated with CVD. Among potentially transient 'state' factors, depression has repeatedly been found to predict CVD independently of the traditional CVD risk factors (Hamieh et al., 2019; Nicholson et al., 2006; Van der Kooy et al., 2007; Wu and Kling, 2016; Wulsin and Singal, 2003). Among presumably stable, 'trait' factors, hostility (i.e. a proneness to experience hostile thoughts or display hostile behaviors) has been the focus of much interest but evidence of an independent association with coronary heart diseases (CHD) is still debated (Chida and Steptoe, 2009; Myrtek, 2001; Newman et al., 2011). However, some hostility features may potentially vary over time (Roberts and DelVecchio, 2000). For instance, a depressive state may increase irritability. In addition, hostile traits could increase the subsequent risk of depression (Nabi et al., 2010). Therefore, current or future depression may potentially explain the association between hostility and CVD. To our knowledge, however, no previous studies explored the association between hostility and CVD incidence in the presence of depression. Hence, a better understanding of this issue may inform the relevance of hostility and depression as independent target for primary prevention of CVD in addition to the traditional CVD risk factors.

The last recent meta-analysis on this topic (Chida and Steptoe, 2009) showed that anger and hostility combined together predict incident CHD (HR of 1.19). However, not only did this meta-analysis merge a psychological 'state' (anger) and a personality 'trait' (hostility), it also used an overall assessment of hostility. Yet, hostility includes a behavioral (or overt) and a cognitive (or covert) component. Various aspects of hostility could lead to CVD through different pathways (e.g. promoting 
coronary atherosclerosis (Matthews et al., 1998; Raikkonen et al., 2004) versus triggering acute events (Dimsdale, 2008; Mittleman et al., 1995)) and therefore need to be studied separately.

Regarding the respective role of depressive symptoms and hostile traits, a previous study conducted by our group suggested that cognitive hostility may substantially explain the association between depression and mortality from natural causes (Lemogne et al., 2010). However, this study could not examine CVD mortality and to our knowledge no other study has reported on CVD incidence. Furthermore, as in many other studies in the field, depression was considered as a time-independent characteristic (i.e. a fixed variable). Since hostile traits could increase the subsequent risk of depression (Nabi et al., 2010), incident depressive symptoms over the follow-up may explain the association between baseline hostility and subsequent CVD. Therefore, longitudinal analyses considering depressive symptoms as a time-dependent characteristic could yield more precise results. Moreover, interventions to alleviate hostility and depression are well-established (McGuire, 2008; Wright et al., 2011) and may be combined with cardiac rehabilitation in secondary prevention (Lavie et al., 2016), but it is not known whether the clinical emphasis should be on hostility, depression, or both.

Taking advantage of the longitudinal assessment of depressive symptoms over 20 years in the French GAZEL cohort study, we examined whether different hostile traits measured at baseline predict incident cardiac events independently of depressive symptoms measured at repeated assessments.

\section{Methods}

\section{Study population}

The GAZEL cohort study (Goldberg et al., 2015) was initiated in 1989 among 20,625 middle-aged (ranged from 35-50 years) workers of the national electricity and gas companies in France (Électricité de FranceGaz de France: EDF-GDF). Briefly, these participants were followed yearly using mailed self-reported questionnaires and human resources databases since 1989. We considered 1993 as our baseline year 
since it was the year in which hostile traits were measured (see below). Among the participants who were alive in 1993 ( $n=20,488$ ), we first excluded those who did not complete the Center for Epidemiologic Studies-Depression Scale (CES-D) at baseline $(n=5,595)$. Compared to volunteers who did not complete the CES-D at baseline, those who did were older, more physically inactive, more likely to have a higher occupational grade and to be male as well as less likely to report having the following diseases: diabetes, dyslipidemia and hypertension (all $P<0.05$ ). Second, we excluded missing data on covariates at baseline (occupational grade, family history of CHD, physical activity, smoking status, body mass index (BMI), diabetes, dyslipidemia and hypertension) $(n=4,020)$ and participants who were diagnosed with a cardiac event before $1994(n=332)$. Compared to individuals with missing data, those with no missing data were older $(P=0.01)$, more likely to be non-obese $(P=0.001)$, non-smokers $(P=0.01)$, to have a higher occupational grade $(P=0.01)$, to be male $(P=0.01)$, less likely to report having depressive symptoms $(P=0.01)$ and had lower levels of hostile traits $(P=0.01)$. Among these 10,541 individuals, we included only those with complete data on the Buss and Durkee Hostility Inventory (BDHI) measured in 1993. Therefore, 10,304 participants were included and followed prospectively for incidence of cardiac events.

The study was approved by the National Institute of Health and Medical Research (INSERM) and the National Data Protection Authority (CNIL) committees. The individuals had given their written consent.

\section{Assessment of incident cardiac events}

All volunteers were followed from January 1, 1994 to December 31, 2014 for cardiac events. To identify the events that occurred before retirement, diagnoses were relied on a registry at the EDF-GDF's medical department (Chevalier et al., 2001) and were medically validated. To identify the events that occurred after retirement, diagnoses came from a systematic validation of each self-reported cardiac event 
through a survey that started in 2009. In each yearly self-administrated questionnaire, the volunteers were asked if they had been hospitalized/diagnosed in the past 12 months with many diseases including cardiac events (i.e. myocardial infarction, angina pectoris, heart failure, cardiac arrhythmia). Volunteers who reported at least one of these cardiac events were asked whether they consent for a detailed investigation including the transmission of their medical records (i.e. procedures and diagnostic tests) and a contact with their physician. All incident cardiac events were validated by two trained independent investigators, either by the use of the volunteers' radiological results, or on a combination of clinical assessments, biological and electrocardiographic tests. Finally, only validated cardiac events were used in our analyses. In sensitivity analyses, we also considered fatal CHD events that were retrieved from the French national cause-of-death registry from January 1, 1994-December 31, 2014. This registry coded the cases relying on the International Classification of Diseases $9^{\text {th }}$ version codes $(410-414)$ and $10^{\text {th }}$ version codes (120-125) (Lemogne et al., 2017).

\section{Assessment of hostility}

Hostility was assessed at baseline in 1993 using the BDHI (Buss and Durkee, 1957), a questionnaire composed of 75 true-false questions, validated in French in a random sample of 408 GAZEL cohort participants (Consoli et al., 1993). It includes eight subscales such as assault, verbal aggression, indirect hostility, irritability, negativism, resentment, suspicion, and guilt tendency. The overall hostility is the sum of all these subscales excluding "guilt tendency". Two overarching factors were identified: behavioral hostility and cognitive hostility. Behavioral hostility included "assault", "verbal aggression" and "indirect hostility", whereas cognitive hostility included "resentment" and "suspicion" (Consoli et al., 1993; Suarez and Williams, 1990). We considered overall (Cronbach's $\alpha=0.88)$, cognitive (Cronbach's $\alpha$ $=0.78$ ) and behavioral (Cronbach's $\alpha=0.80$ ) hostility scores as well as two subscales: irritability (Cronbach's $\alpha=0.70$ ) and negativism (Cronbach's $\alpha=0.53$ ). The overall hostility score has been found to 
have a high 3-months test-retest reliability $(r=0.87)$ (Consoli et al., 1993). Overall hostility and the hostile traits of interest (cognitive hostility, behavioral hostility, irritability and negativism) were used as continuous variables taking their interquartile range (IQR) as the unit of measurement (difference between the $75^{\text {th }}$ and $25^{\text {th }}$ percentiles).

\section{Assessment of depressive symptoms}

Depressive symptoms were assessed using the French validated version of CES-D, a scale of 20 items of depressive symptoms (Fuhrer and Rouillon, 1989) administered 7 times, at baseline (1993), 1996, 1999, 2002, 2005, 2008 and 2012. The CES-D questionnaire has a high internal consistency (Cronbach's $\alpha$ from 0.8 to 0.9 ) in community samples (Radloff, 1977). This screening test measures sadness, loss of interest, appetite, sleep, thinking, guilt, fatigue, agitation and suicidal thoughts in the previous week on a scale of 0 ( $<1$ day) to 3 ( 5 to 7 days). To ease the interpretation of the results, we first used a binary measure of depressive symptoms. As in previous studies from the GAZEL cohort, depressive symptoms were defined based on a CES-D score meeting the validated cutoff of 17 in men and 23 in women (Fuhrer and Rouillon, 1989). However, since sole reliance on a binary cutoff may be statistically unsafe (MacCallum et al., 2002), sensitivity analyses were performed with the CES-D score as continuous variable.

\section{Assessment of covariates}

Socio-demographics such as age $(39-45 ; 46-49 ; 50-54)$, sex and occupational grade (low: clerks or manual workers; intermediate: technical workers or first-line supervisors; high: engineers, managers or lawyers) were collected from the EDF-GDF human resources department in 1993. Family history of CHD (no; yes: having at least one parent with CHD before the age of 60) was self-reported in 1993.

Modifiable CVD risk factors were self-reported annually on questionnaires between 1993 and 2013 and included physical activity (yes: occasional or regular activity; no), smoking status (non-smokers; 
ex-smokers; current smokers) and BMI $\left(<25 ; \geq 25\right.$ and $<30 ; \geq 30 \mathrm{~kg} / \mathrm{m}^{2}$, based on the participants' height and weight), diabetes (yes; no), dyslipidemia (yes; no) and hypertension (yes; no). Diabetes, dyslipidemia and hypertension were assessed using the following two questions: 'In the preceding 12 months, did you suffer from the diseases listed below?' and 'Among those diseases, which are the ones that occurred in the preceding 12 months?'

\section{Statistical analysis}

All $P$ values were two-sided $(\alpha=0.05)$. Analyses were undertaken using the SAS system software version 9.4 and the SAS-derived JMP discovery software 12 (SAS Institute, Inc., Cary, NC). Each participant contributed person-time from the date of completion of the baseline GAZEL study questionnaire until a) a cardiac event, b) the last follow-up questionnaire, c) death, or d) end of the study (December 31, 2014), whichever came first. If a participant had a validated non-fatal cardiac event before the end of the study, he or she contributed for non-fatal events, even if he or she died later of a CHD event.

We computed descriptive estimates of socio-demographics, family history of CHD and modifiable CVD risk factors by depressive symptoms and overall hostility at baseline using Chi-square and ANOVA tests for categorical and continuous variables respectively.

We used Cox proportional hazards models to calculate the hazard ratios (HR) and 95\% confidence intervals $(\mathrm{Cl})$ of hostile traits for incident cardiac events. In Model 1, we adjusted for sociodemographics and family history of CHD at baseline. Then, considering that depression is associated with several modifiable CVD risk factors (Gaye et al., 2016; Meneton et al., 2016) and to explore the extent to which hostile traits are associated with cardiac events independently of depressive symptoms, Model 2 was further adjusted for time-dependent depressive symptoms as a binary variable. In addition, the CESD score was used as a continuous score (taking IQR as unit) in sensitivity analyses. Finally, in Model 3, we adjusted for time-dependent modifiable CVD risk factors in addition to the covariates in Model 2. 


\section{Results}

The baseline characteristics of the 10,304 GAZEL participants included in the analyses are displayed in Table 1. A total of 2,324 participants (22.6\%) had depressive symptoms (binary measure) at study baseline (Table 2). Sex, occupational grade, physical activity, smoking status, dyslipidemia and hypertension were significantly associated with depressive symptoms (binary measure). Whereas, age, sex, occupational grade, family history of CHD, physical activity, smoking status, BMI, diabetes, dyslipidemia and hypertension were significantly associated with overall hostility.

After 20 years of follow-up (mean of 19.2 with a standard deviation of 4.1 years), 581 (5.6\%) incident, non-fatal cases of cardiac events were identified. These cardiac events included 464 CHDrelated events (i.e. 159 angina pectoris and 305 myocardial infarction cases) and 117 other cardiac events (e.g. heart failure, cardiac arrhythmia). Participants with incident cardiac events were significantly older, more likely to be male, to have a high occupational grade, family history of CHD and a BMI $\geq 30$, to smoke and to report diabetes, dyslipidemia and hypertension at baseline $(p<0.05)$.

Adjusting for socio-demographics and family history of CHD, (Model 1), among the hostile traits at baseline, only irritability was associated with incident cardiac events ( $\mathrm{HR} 1.16,95 \% \mathrm{Cl} 1.02-1.32$ ) (Table 3). However, this association was no longer significant when further adjusting for time-dependent depressive symptoms (binary measure) in Model 2 ( $\mathrm{HR} 0.88,95 \% \mathrm{Cl} 0.68-1.15$ ). Similar findings were obtained when using the CES-D score as a continuous measure of depressive symptoms (HR for irritability in Model 2: $0.88,95 \% \mathrm{Cl}$ 0.67-1.16). In contrast, depressive symptoms (binary measure) were significantly associated with incident cardiac events, whatever the hostile trait included in the model, with HRs ranging from 1.51-1.63 in Model 2 and from 1.40-1.49 in Model 3.

Since irritability was no longer associated with incident cardiac events when accounting for timedependent depressive symptoms (binary measure), we checked whether it could be explained by an 
association between irritability and the risk of depressive symptoms (binary measure). But, given the small effect size of the association between irritability and cardiac events in Model 1 , we did not run formal mediation analysis.

Finally, 75 participants died due to a fatal CHD event during the follow-up. Sensitivity analyses that added these CHD fatal events to the non-fatal cardiac events yielded similar results, irritability being weakly but significantly associated with cardiac events in Model 1 (HR: 1.11,95\%Cl 1.01-1.36) only, while depressive symptoms (binary measure) remaining a robust predictor of cardiac event in Model 3 (HR: $1.41,95 \% \mathrm{Cl} 1.05-2.07)$.

\section{Discussion}

We aimed to study whether different hostile traits measured at baseline predict incident cardiac events independently of depressive symptoms measured at repeated assessments over 20 years of follow-up. After adjustment for socio-demographics and family history of $\mathrm{CHD}$, irritability was the only hostile trait significantly associated with incident cardiac events. However, when adjusting for depressive symptoms, either as a binary or a continuous measure, this association was no longer statistically significant. In contrast, depressive symptoms remained significant predictors of incident cardiac events, whatever the hostile trait included in the model, even when adjusting for modifiable risk factors. Overall, our results suggest that incident depressive symptoms may explain the association between irritability and incident cardiac events.

Our study has several strengths. To our knowledge, this is the first study that investigated several hostile traits in relation to incident cardiac events. The use of validated cardiac events instead of selfreported cases ensures the reliability of our results. The analysis considered a wide range of longitudinally assessed CVD risk factors over a 20-year follow-up. It is also the first study that assesses the association between hostility and incident cardiac events using time-dependent covariates. In 
addition, the GAZEL population is a socioeconomically diverse population across all the regions of France. The GAZEL cohort study has a very low loss to follow-up rate and a long follow-up duration (Goldberg et al., 2015).

However, the present study has several limitations. The GAZEL population represents only middle-aged persons employed at baseline; hence, it does not represent the general population, particularly those who are unemployed. In addition, relying on complete cases may have resulted in selection bias due to missing data. In particular, participants were less likely to be depressed and had lower levels of hostile traits than non-participants which might have reduced the statistical power of the study. The CES-D scale assesses depressive symptoms only and not major depression; but depressive symptoms, and not only major depression, were associated with incident cardiac events as well (Hamieh et al., 2019; Meneton et al., 2016). The CVD risk factors were assessed using self-reports, but their strong associations with cardiac events, as shown in previous reports (Lemogne et al., 2017), were consistent with previous studies, which provide external validity. Since cardiac events were validated, the likelihood of false-positives is reduced. Although sensitivity analyses including CHD fatal events yielded similar results, we cannot rule out that some false negatives may have occurred, but the impact of misclassification on our findings is probably limited. Finally, hostile traits were only assessed once at baseline. Although personality is defined as stable over time, empirical evidence suggests that some dimensions may substantially vary over long period of time (Roberts and DelVecchio, 2000). Future studies may also consider hostility as potentially time-varying, focusing on irritable mood as a state rather than a trait. However, should irritability have varied according to depression, it may have remained associated with cardiac events but, in this case, would have been interpreted as a mere symptom of depression, leading to similar conclusions.

The first finding of our study is indeed that irritability was the only hostile trait to show an association with incident cardiac events. This finding confirms that considering hostility as a multi- 
faceted construct is important. It may explain the literature inconsistencies regarding hostility as a whole (Chida and Steptoe, 2009). Since the seminal study of Friedman and Rosenman (Friedman and Rosenman, 1959), several studies showed a significant association between irritability and CVD (Fava et al., 2017; Mendes de Leon, 1992; Perlis et al., 2005; Porcelli and Guidi, 2015; Siegman et al., 2000; Sirri et al., 2012; Sonino et al., 2004). Beside irritability as a trait, the prevalence of irritable mood, as assessed with the Diagnostic Criteria for Psychosomatic Research (DCRP), may be as high as $10-15 \%$ in medical patients, including patients with myocardial infarction (Fava et al., 2017). Another study showed that the rate of DCPR irritable mood was higher in patients with vs. without cardiac disease, irrespective of Type A behavior (Sirri et al., 2012). Therefore, some authors emphasized that physicians should consider depressive mood and irritability as CVD risk factors (Ottolini et al., 2005).

The second important finding of our study is that irritability no longer predicted incident cardiac events when adjusting for depressive symptoms. This was observed even before adjustment for modifiable CVD risk factors, suggesting that depressive symptoms could constitute a preventive target on its own. In contrast, irrespective of the hostile trait considered in the model, depressive symptoms were robustly associated with incident cardiac events, independently of CVD risk factors. In a previous study by our group (Lemogne et al., 2010), depressive symptoms were associated with natural mortality after adjustment for non-modifiable CVD risk factors, but this association disappeared after further adjusting for cognitive hostility, which became the only significant predictor of mortality. Beyond the differences in the outcomes, these discrepancies might be merely explained by the fact that the present study modelled depressive symptoms as a time-independent variable. Interestingly, our results obtained in a population-based study in which participants were free of CVD at baseline were consistent with those of a study among patients with CHD where depressive symptoms explained most of the mortality risk after anxiety and hostility measures were included (Kachur et al., 2016). 
In conclusion, incident depressive symptoms might explain the prospective association between irritability and cardiac events. Therefore, this study, which is the first to investigate several hostile traits and depressive symptoms in relation to incident cardiac events, has clinical implications. Specifically, efforts aiming at reducing the risk of cardiac events associated with hostility should focus on depressive symptoms as a potentially modifiable factor. These efforts should also be informed by further research exploring the mechanisms linking depressive symptoms with CVD risk that could be targeted in preventive interventions. Finally, these results may constitute an impetus for reanalyzing old datasets considering depression as a time-varying state.

\section{Acknowledgements}

We would like to thank Sébastien Bonenfant for helping us in the data management. 


\section{References}

Buss, A.H., Durkee, A., 1957. An inventory for assessing different kinds of hostility. J Consult Psychol 21, 343-349.

Chevalier, A., Zins, M., Godard, C., Morin, J., Jourdain, V., Francois, F., Lambrozo, J., Goldberg, M., Ducimetiere, P., 2001. [ A registry of ischaemic cardiopathies among active workers at Electricite de France-Gaz de France. Program development and first results]. Rev Epidemiol Sante Publique 49, 51-60.

Chida, Y., Steptoe, A., 2009. The association of anger and hostility with future coronary heart disease: a meta-analytic review of prospective evidence. J Am Coll Cardiol 53, 936-946.

Consoli, S.M., Cordier, S., Ducimetiere, P., 1993. [Validation of a personality questionnaire designed for defining sub-groups at risk for ischemic cardiopathy or cancer in the Gazel cohort]. Rev Epidemiol Sante Publique 41, 315-326.

Dimsdale, J.E., 2008. Psychological stress and cardiovascular disease. J Am Coll Cardiol 51, 12371246.

Ezzati M, Hoorn SV, Rodgers A, Lopez AD, Mathers CD, CJ, M., 2003. Comparative Risk Assessment Collaborating Group. Estimates of global and regional potential health gains from reducing multiple major risk factors. Lancet 362, 271-280.

Ezzati M, Lopez AD, Rodgers A, CJL, M., 2004. Comparative Quantification of Health Risks. Global and Regional Burden of Disease Attributable to Selected Major Risk Factors, World Health Organization, Geneva, Switzerland.

Ezzati M, Lopez AD, Rodgers A, Vander Hoorn S, CJ, M., 2002. Comparative Risk Assessment Collaborating Group. Selected major risk factors and global and regional burden of disease. Lancet 360, 1347-1360.

Ezzati, M., Henley, S.J., Thun, M.J., Lopez, A.D., 2005. Role of smoking in global and regional cardiovascular mortality. Circulation 112, 489-497.

Fava, G.A., Cosci, F., Sonino, N., 2017. Current Psychosomatic Practice. Psychother Psychosom 86, 13-30.

Friedman, M., Rosenman, R.H., 1959. Association of specific overt behavior pattern with blood and cardiovascular findings; blood cholesterol level, blood clotting time, incidence of arcus senilis, and clinical coronary artery disease. J Am Med Assoc 169, 1286-1296.

Fuhrer, R., Rouillon, F., 1989. La version française de l'échelle CES-D (Center for Epidemiologic Studies-Depression Scale). Description et traduction de l'échelle d'autoévaluation [The French version of the CES-D (Center for Epidemiologic Studies-Depression Scale)]. Psychiatrie \& Psychobiologie 4, 163-166.

Gaye, B., Prugger, C., Perier, M.C., Thomas, F., Plichart, M., Guibout, C., Lemogne, C., Pannier, B., Boutouyrie, P., Jouven, X., Empana, J.P., 2016. High level of depressive symptoms as a barrier to reach an ideal cardiovascular health. The Paris Prospective Study III. Sci Rep 6, 18951.

Goldberg, M., Leclerc, A., Zins, M., 2015. Cohort Profile Update: The GAZEL Cohort Study. Int J Epidemiol 44, 77-77g.

Hamieh, N., Meneton, P., Wiernik, E., Limosin, F., Zins, M., Goldberg, M., Melchior, M., Lemogne, C., 2019. Depression, treatable cardiovascular risk factors and incident cardiac events in the Gazel cohort. Int J Cardiol 284, 90-95. 
Kachur, S., Menezes, A.R., De Schutter, A., Milani, R.V., Lavie, C.J., 2016. Significance of Comorbid Psychological Stress and Depression on Outcomes After Cardiac Rehabilitation. Am J Med 129, 1316-1321.

Lavie, C.J., Menezes, A.R., De Schutter, A., Milani, R.V., Blumenthal, J.A., 2016. Impact of Cardiac Rehabilitation and Exercise Training on Psychological Risk Factors and Subsequent Prognosis in Patients With Cardiovascular Disease. Can J Cardiol 32, S365-S373.

Lemogne, C., Meneton, P., Wiernik, E., Quesnot, A., Consoli, S.M., Ducimetiere, P., Nabi, H., Empana, J.P., Hoertel, N., Limosin, F., Goldberg, M., Zins, M., 2017. When Blue-Collars Feel Blue: Depression and Low Occupational Grade as Synergistic Predictors of Incident Cardiac Events in Middle-Aged Working Individuals. Circ Cardiovasc Qual Outcomes 10.

Lemogne, C., Nabi, H., Zins, M., Cordier, S., Ducimetiere, P., Goldberg, M., Consoli, S.M., 2010. Hostility may explain the association between depressive mood and mortality: evidence from the French GAZEL cohort study. Psychother Psychosom 79, 164-171.

MacCallum, R.C., Zhang, S., Preacher, K.J., Rucker, D.D., 2002. On the practice of dichotomization of quantitative variables. Psychol Methods 7, 19-40.

Matthews, K.A., Owens, J.F., Kuller, L.H., Sutton-Tyrrell, K., Jansen-McWilliams, L., 1998. Are hostility and anxiety associated with carotid atherosclerosis in healthy postmenopausal women? Psychosom Med 60, 633-638.

McGuire, J., 2008. A review of effective interventions for reducing aggression and violence. Philos Trans R Soc Lond B Biol Sci 363, 2577-2597.

Mendes de Leon, C.F., 1992. Anger and impatience/irritability in patients of low socioeconomic status with acute coronary heart disease. J Behav Med 15, 273-284.

Meneton, P., Lemogne, C., Herquelot, E., Bonenfant, S., Larson, M.G., Vasan, R.S., Menard, J., Goldberg, M., Zins, M., 2016. A Global View of the Relationships between the Main Behavioural and Clinical Cardiovascular Risk Factors in the GAZEL Prospective Cohort. PLoS One 11, e0162386.

Mittleman, M.A., Maclure, M., Sherwood, J.B., Mulry, R.P., Tofler, G.H., Jacobs, S.C., Friedman, R., Benson, H., Muller, J.E., 1995. Triggering of acute myocardial infarction onset by episodes of anger. Determinants of Myocardial Infarction Onset Study Investigators. Circulation 92, 17201725.

Myrtek, M., 2001. Meta-analyses of prospective studies on coronary heart disease, type A personality, and hostility. Int J Cardiol 79, 245-251.

Nabi, H., Singh-Manoux, A., Ferrie, J.E., Marmot, M.G., Melchior, M., Kivimaki, M., 2010. Hostility and depressive mood: results from the Whitehall II prospective cohort study. Psychological medicine 40, 405-413.

Newman, J.D., Davidson, K.W., Shaffer, J.A., Schwartz, J.E., Chaplin, W., Kirkland, S., Shimbo, D., 2011. Observed hostility and the risk of incident ischemic heart disease: a prospective population study from the 1995 Canadian Nova Scotia Health Survey. J Am Coll Cardiol 58, 12221228.

Nicholson, A., Kuper, H., Hemingway, H., 2006. Depression as an aetiologic and prognostic factor in coronary heart disease: a meta-analysis of 6362 events among 146538 participants in 54 observational studies. Eur Heart J 27, 2763-2774. 
Ottolini, F., Modena, M.G., Rigatelli, M., 2005. Prodromal symptoms in myocardial infarction. Psychother Psychosom 74, 323-327.

Perlis, R.H., Fraguas, R., Fava, M., Trivedi, M.H., Luther, J.F., Wisniewski, S.R., Rush, A.J., 2005. Prevalence and clinical correlates of irritability in major depressive disorder: a preliminary report from the Sequenced Treatment Alternatives to Relieve Depression study. J Clin Psychiatry 66, 159-166; quiz 147, 273-154.

Porcelli, P., Guidi, J., 2015. The Clinical Utility of the Diagnostic Criteria for Psychosomatic Research: A Review of Studies. Psychother Psychosom 84, 265-272.

Radloff, L.S., 1977. The CES-D Scale: A Self-Report Depression Scale for Research in the General Population. Applied Psychological Measurement 1, 385-401.

Raikkonen, K., Matthews, K.A., Sutton-Tyrrell, K., Kuller, L.H., 2004. Trait anger and the metabolic syndrome predict progression of carotid atherosclerosis in healthy middle-aged women. Psychosom Med 66, 903-908.

Roberts, B.W., DelVecchio, W.F., 2000. The rank-order consistency of personality traits from childhood to old age: a quantitative review of longitudinal studies. Psychol Bull 126, 3-25. Siegman, A.W., Townsend, S.T., Civelek, A.C., Blumenthal, R.S., 2000. Antagonistic behavior, dominance, hostility, and coronary heart disease. Psychosom Med 62, 248-257.

Sirri, L., Fava, G.A., Guidi, J., Porcelli, P., Rafanelli, C., Bellomo, A., Grandi, S., Grassi, L., Pasquini, P., Picardi, A., Quartesan, R., Rigatelli, M., Sonino, N., 2012. Type A behaviour: a reappraisal of its characteristics in cardiovascular disease. Int J Clin Pract 66, 854-861.

Sonino, N., Navarrini, C., Ruini, C., Ottolini, F., Paoletta, A., Fallo, F., Boscaro, M., Fava, G.A., 2004. Persistent psychological distress in patients treated for endocrine disease. Psychother Psychosom 73, 78-83.

Suarez, E.C., Williams, R.B., Jr., 1990. The relationships between dimensions of hostility and cardiovascular reactivity as a function of task characteristics. Psychosom Med 52, 558-570. Tzoulaki, I., Elliott, P., Kontis, V., Ezzati, M., 2016. Worldwide Exposures to Cardiovascular Risk Factors and Associated Health Effects: Current Knowledge and Data Gaps. Circulation 133, 23142333.

Van der Kooy, K., van Hout, H., Marwijk, H., Marten, H., Stehouwer, C., Beekman, A., 2007. Depression and the risk for cardiovascular diseases: systematic review and meta analysis. Int J Geriatr Psychiatry 22, 613-626.

World Health Organization, 2017, May 17. Cardiovascular diseases (CVDs). World Health Organization.

Wright, L.B., Gregoski, M.J., Tingen, M.S., Barnes, V.A., Treiber, F.A., 2011. Impact of Stress Reduction Interventions on Hostility and Ambulatory Systolic Blood Pressure in African American Adolescents. J Black Psychol 37, 210-233.

Wu, Q., Kling, J.M., 2016. Depression and the Risk of Myocardial Infarction and Coronary Death: A Meta-Analysis of Prospective Cohort Studies. Medicine 95, e2815.

Wulsin, L.R., Singal, B.M., 2003. Do depressive symptoms increase the risk for the onset of coronary disease? A systematic quantitative review. Psychosom Med 65, 201-210. 
Table 1. Baseline characteristics of 10,304 participants in the GAZEL cohort study, 1994-2014

\begin{tabular}{|c|c|}
\hline \multicolumn{2}{|l|}{ Discrete variables } \\
\hline \multicolumn{2}{|l|}{ Age } \\
\hline $39-45, \%$ & 33.0 \\
\hline $46-49, \%$ & 33.0 \\
\hline $50-54, \%$ & 34.0 \\
\hline \multicolumn{2}{|l|}{ Sex } \\
\hline Female, \% & 25.4 \\
\hline Male, \% & 74.6 \\
\hline \multicolumn{2}{|l|}{ Occupational grade } \\
\hline Low, \% & 11.7 \\
\hline Intermediate, \% & 55.5 \\
\hline High, \% & 32.8 \\
\hline \multicolumn{2}{|l|}{ Family history of CHD } \\
\hline No, $\%$ & 88.0 \\
\hline Yes, \% & 12.0 \\
\hline \multicolumn{2}{|l|}{ Physical activity } \\
\hline No, $\%$ & 31.2 \\
\hline Yes, \% & 68.9 \\
\hline \multicolumn{2}{|l|}{ Smoking status } \\
\hline Non-smokers, \% & 43.8 \\
\hline Ex-smokers, \% & 37.4 \\
\hline Smokers, \% & 18.7 \\
\hline \multicolumn{2}{|l|}{ Body mass index } \\
\hline$<25, \%$ & 53.7 \\
\hline$\geq 25$ and $<30, \%$ & 40.4 \\
\hline$\geq 30, \%$ & 5.9 \\
\hline \multicolumn{2}{|l|}{ Diabetes } \\
\hline No, $\%$ & 98.5 \\
\hline Yes, \% & 1.5 \\
\hline \multicolumn{2}{|l|}{ Dyslipidemia } \\
\hline No, $\%$ & 84.2 \\
\hline Yes, \% & 15.8 \\
\hline \multicolumn{2}{|l|}{ Hypertension } \\
\hline No, $\%$ & 90.6 \\
\hline Yes, \% & 9.4 \\
\hline \multicolumn{2}{|l|}{ Depressive symptoms* } \\
\hline No, $\%$ & 77.4 \\
\hline Yes, \% & 22.6 \\
\hline \multicolumn{2}{|l|}{ Continuous variables } \\
\hline Mean (SD) overall hostility & 28.1 (9.9) \\
\hline
\end{tabular}


Table 2. Baseline associations between depressive symptoms or hostility and the characteristics of 10,304 participants in the GAZEL cohort study, 1994-2014

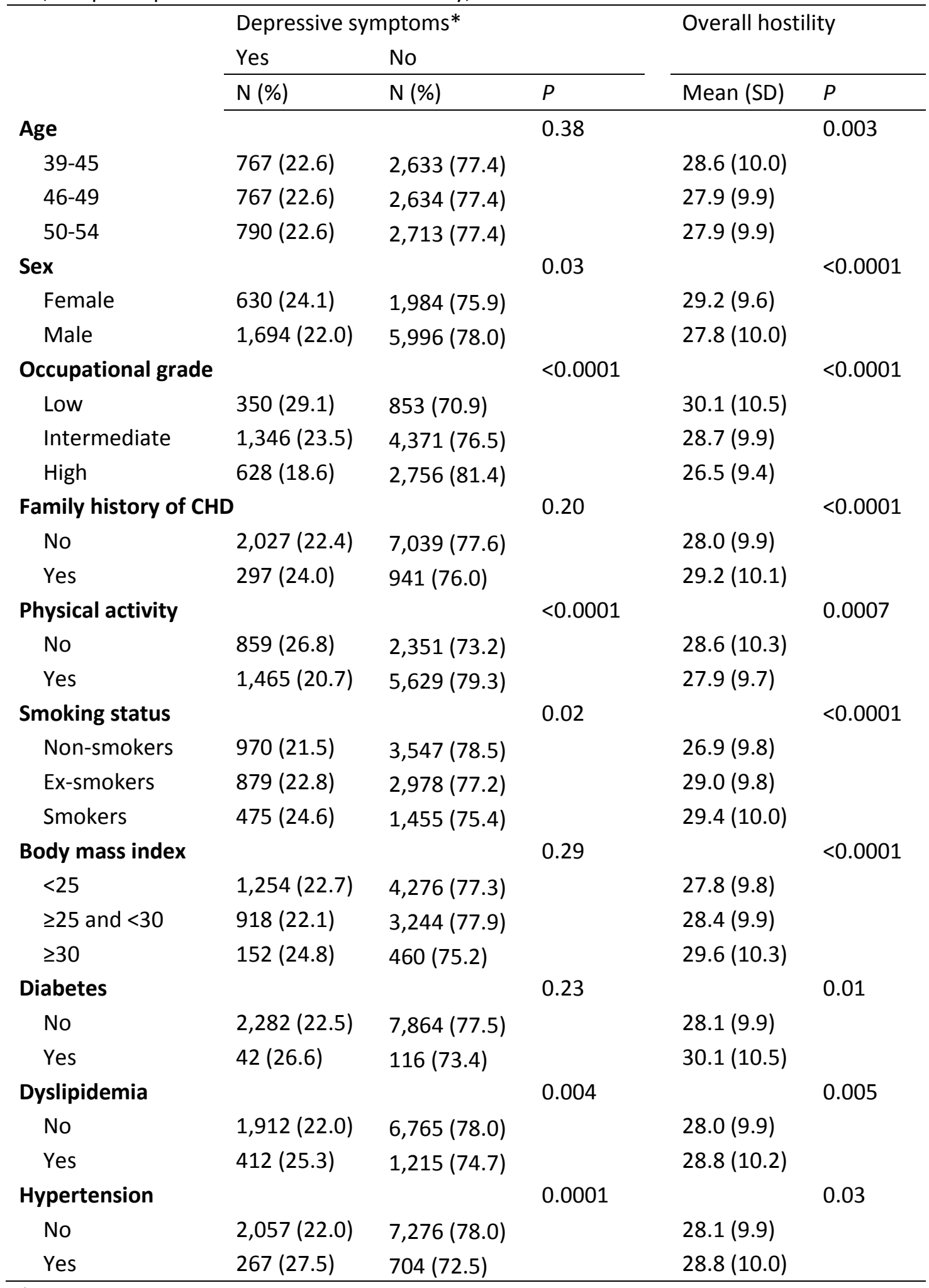

*CES-D score $\geq 17$ in men or $\geq 23$ in women.

ANOVA and Chi-square tests were computed for continuous and categorical variables respectively. 
Table 3. Association between a) hostile traits and b) depressive symptoms and non-fatal cardiac event in the GAZEL cohort study, 1994-2014 (multivariable hazard ratios, HRs and 95\% confidence intervals, $\mathrm{Cl}$ ).

\begin{tabular}{|c|c|c|c|}
\hline & Model $1 \infty$ & Model 2† & Model 3§ \\
\hline & $\mathrm{HR}(95 \% \mathrm{Cl})$ & $\mathrm{HR}(95 \% \mathrm{Cl})$ & $\mathrm{HR}(95 \% \mathrm{Cl})$ \\
\hline Depressive symptoms* & - & $1.51(1.17-1.96)$ & $1.40(1.04-1.90)$ \\
\hline Overall hostility** [IQR:35-21] & $1.11(0.99-1.25)$ & $0.93(0.73-1.19)$ & $0.87(0.65-1.17)$ \\
\hline Depressive symptoms* & - & $1.53(1.18-1.98)$ & $1.42(1.05-1.92)$ \\
\hline Cognitive hostility** [IQR:8-3] & $1.03(0.91-1.16)$ & $0.92(0.72-1.17)$ & $0.74(0.55-1.00)$ \\
\hline Depressive symptoms* & - & $1.57(1.23-2.01)$ & $1.49(1.12-1.99)$ \\
\hline Behavioral hostility** [IQR:18-11] & $1.09(0.98-1.22)$ & $0.97(0.78-1.21)$ & $0.96(0.74-1.25)$ \\
\hline Depressive symptoms* & - & $1.58(1.23-2.04)$ & $1.47(1.09-1.98)$ \\
\hline Irritability** [IQR:8-4] & $1.16(1.02-1.32)$ & $0.88(0.68-1.15)$ & $0.87(0.63-1.20)$ \\
\hline Depressive symptoms* & - & $1.63(1.28-2.08)$ & $1.49(1.12-1.98)$ \\
\hline Negativism** [IQR:3-1] & $1.07(0.94-1.22)$ & $1.06(0.83-1.37)$ & $1.19(0.88-1.61)$ \\
\hline
\end{tabular}

Number of cardiac events: 581

IQR interquartile range (i.e. the interval between the $25^{\text {th }}$ and the $75^{\text {th }}$ percentile)

*CES-D score $\geq 17$ in men or $\geq 23$ in women.

**expressed as an increase of 1 IQR

$\infty$ Adjusted for age, sex, occupational grade and family history of CHD.

† Model $1+$ additionally adjusted for time-dependent depressive symptoms.

$\S$ Model 2+ additionally adjusted for time-dependent physical activity, smoking status, BMI, diabetes, dyslipidemia and hypertension. 